\title{
Evaluation of the effect of the cumulative centrifugation force on the characteristics and the tissue regeneration potential of the platelet rich fibrin: a clinical and laboratory study
}

\section{Original Article}

\author{
Mosaad Abdaljawwad Khalifah
}

Department of Oral and Maxillofacial Surgery, Faculty of Dentistry, Kafr ElSheikh

University, Kafr ElSheikh, Egypt

\begin{abstract}
Background: Although the platelet-rich fibrin (PRF) gained a considerable popularity as a tissue regeneration technique to reconstruct the surgical defets, the effect of the cumulative centrifugation force (in terms of speed and time) on its regeneration potential is unclear.

Material and Methods: Ten patients with maxillary surgical defects were randomly and equally divided into two groups where in the study group, the centrifugation speed and time were $1500 \mathrm{rpm}$ and 18 minutes respectively and in the control group, these centrifugation parameters were $2700 \mathrm{rpm}$ and 12 minutes on order. For each patient, the surgical defect was packed with the clots and an extra clot was assigned for histologic, immunohistochemical and histomorphometric examination.

Results: The laboratory results exhibited that the slower but longer duration centrifugation resulted in a looser fibrin clot with larger interfiber spaces and deeper penetration of neutrophilic granulocytes. In both groups, the other cells (except for platelets which were distributed through out the clot) were confined to the proximal $1 / 3-1 / 4$ of the clot. But that difference failed to impact the regeneration potential on the clinical level as assessed by bone formation scale and density changes.

Conclusion: Although changing the comulative centrifugation force resulted in deeper penetration of granulocytes, the regenerative potential did not enhance, perhaps due to the complex nature of the regeneration process where one element alone cannot alter the regeneration potential.
\end{abstract}

Key Words: Bone, Cell distribution, Cumulative centrifugation force, PRF and Regeneration.

Received: 06 November 2020, Accepted: 19 January 2021.

Corresponding Author: Mosaad Abdaljawwad Abdalmawla Khalifah, Department of Oral and Maxillofacial Surgery, Faculty of Dentistry, Kafr ElSheikh University, Kafr ElSheikh, Egypt, Tel.: +20452738074, Mobile: +201008603084, E-mail: mosaad_khalifa@den.kfs.edu.eg.

ISSN: 2090-097X, July 2020, Vol. 11, No. 3

\section{INTRODUCTION}

The treatment of surgical defects might pose a major challenge to the surgeon whether on the level of the hard or the soft tissues ${ }^{[1]}$. Tissues regeneration based on a material induced tissue reaction that acted as a gap bridging tissue to the surgical defect has gained a wide consideration ${ }^{[2]}$. The techniques requiring a precultivation and special cell manipulation are more difficult, technique sensitive, and time consuming than those could be widely applied on the clinical level ${ }^{[3]}$. Platelet rich plasma (PRP) was the first clinical level scaffold tissue regenerating biomaterial produced from the human autologous blood ${ }^{[4]}$. However, as its formation required the addition of bovine serum and anticoagulant, the call for more clinically streamlining strategies lead to the introduction of platelet rich fibrin $(\mathrm{PRF})^{[5]}$. Choukroun's PRF provides the surgeon with a cell loaded three dimensional fibrin network contains growth factors. The wide variety of the entrapped blood cells included platelets, stem cells, momocytes, neutrophilic granulocytes and B- and T- lymphocytes ${ }^{[6,7]}$. Despite PRF was then applied in various studied ${ }^{[8-10]}$, the effect of the cumulative centrifugation force (in terms of speed and time) on the fibrin properties and the cell distribution pattern within the fibrin matrix scaffold is not clear and if it has an effect, the capability to lead to a different clinical level regeneration potential is unclear as well. Thus, the current study aimed at evaluating the effect of the cumulative centrifugation force on the characteristics of the PRF (in terms of the fibrin network and the cell distribution pattern within the matrix) and on the tissue regeneration potential.

\section{MATERIALS AND METHODS}

Sample size:

The sample size was determined to be ten patients equally divided into two groups using power analysis at $\alpha=0.05, \beta=0.2$, enrollment ratio $=1$, a continuous primary endpoint and assumed penetration of 0.25 and 0.68 for the control and study groups respectively. A post hoc power analysis showed a $79.8 \%$ level of power at $\alpha=0.05$ and 5 patients allocated for each group.

\section{Subjects:}

A total of ten patients with surgical bony defects in the anterior maxilla not exceeding $2 * 3 \mathrm{~cm}^{2}$ from the outpatient department of Oral and Maxillofacial Surgery department 
within the age range of 18 to 45 years were included in the current study. The status of being otherwise healthy patient was an inclusion criterion. Exclusion criteria included any disease could affect the healing, hormonal disturbances, any bone or connective tissue disease and bleeding or coagulation disorders. Patients were randomly divided into two equal groups using a computer permuted block stratified randomization generator (randomization.com).

\section{Study design:}

For group I patients (the study group), a sufficient volume of the venous blood were withdrawn from the patient's antecubital vein into a sterile glass tube which was then immediately placed in the preprogrammed centrifuge device adjusted on $1500 \mathrm{rpm}$ and 18 minutes centrifugation time. The blood amount was estimated according to the surgical defect size with an extra $10 \mathrm{~mL}$ of blood to produce the PRF sample assigned for microscopic (immunehistochemical and histomorphometric analysis). The clots assigned for clinical use were carefully retrieved from the tubes and the red blood cell (RBC) fraction was removed with a scissors. The PRFs were then packed in the surgical defect and the flaps were reapproximated. Peri operative photographs were taken. The PRF sample assigned for microscopic examination was also carefully retrieved from the tube and most of the $\mathrm{RBC}$ fraction was removed with a scissors (leaving only about $2 \mathrm{~mm}$ ) and then was sent for Alborg specialized histopathological laboratory, Egypt to be fixed with a $4 \%$ paraformaldehyde solution for 24 hours to be then cut and placed into embedding cassettes along its longitudinal axis. Then the sample was chemically processed into an alcohol series and xylene before being embedded into paraffin.
Ten sections were cut with a rotary microtome with a thickness of 24- $\mu \mathrm{m}$ and affixed on charged glass slabs. Deparaffinization and rehydratation were then performed by sequential immersion in xylene followed by descending concentrations of ethanol. Several histochemical and immunohistochemical staining was carried out as follows. Four samples were stained with hematoxylin and eosin ( $\mathrm{H}$ and $\mathrm{E})$, Masson Goldner trichrome and Giemsa's stains to discriminate the clot components. Histological examination was performed using a light microscope (Nikon Eclipse 80i, Tokyo, Japan) under the high power. Six sections were allocated for immunohistochemical staining to identify the markers (DAKO, Hamburg, Germany) mentioned in Table 1. Following establishing the optimal antibody concentration for each marker, the slides were placed in a rack and incubated for 20 minutes in Tris-EDTA pH 8.0 at $968 \mathrm{C}$ and subsequently were rinsed with a running water to cool before being washed with triss buffered saline (TBS) and transferred to an autostainer (DAKO, Hamburg, Germany). The DAKO EnVision detection system was used. Then the slides were counterstained with hemalun for 30 seconds before being washed with running water. Finally, the slides were cover slipped with water based mounting medium (Quick Mount Aquous, TedPella Inc., Redding, CA) to be examined. Histomorphometric analysis was performed by scanning the slides with the microscope with fitting the automatic scanning table (Prior Scientific, Rockland, Maine). A digital camera (Nikon DS-Fi/1) and a computer with the Nikon NIS Elements AR software, version 4.0 were

used to capture single high-resolution images and then to assemble them to one complete image to generate a total scan. The measurement function of the NIS Elements software was used to measure the total length of the clot and the penetration length of every cell type in the clot.

Table 1: Immunohistochemical markers and their targeted cells:

\begin{tabular}{|c|c|c|c|c|}
\hline Antibody* & Concentration & $\begin{array}{c}\text { Type } \\
\text { (mono or poly) }\end{array}$ & Clone & Targeted cell \\
\hline Anti-CD3 & $1: 200$ & Polyclonal rabbit anti-human & - & T-lymphocytes \\
\hline Anti-CD20cy & $1: 1000$ & Monoclonal mouse anti- human & Clone L26 & B-lymphocytes \\
\hline Anti-CD34 class II & RTU & Monoclonal mouse anti- human & Clone QBend-10 & Stem cells \\
\hline Anti-CD15 & RTU & Monoclonal mouse anti- human & Carb-3 & $\begin{array}{l}\text { Neutrophilic } \\
\text { granulocytes }\end{array}$ \\
\hline $\begin{array}{l}\text { Anti-CD61 platelet } \\
\text { glycoprotein IIIa }\end{array}$ & $1: 500$ & Monoclonal mouse anti- human & $\mathrm{Y} 2 / 51$ & Platelets \\
\hline Anti-CD68 & RTU & Monoclonal mouse anti- human & KP1 & Monocyte \\
\hline
\end{tabular}


For group II patients (the study group), the same procedure was applied with the sole difference to be in the setting of the centrifuge device that were adjusted on 2700 rpm and 12 minutes centrifugation time.

This study complied with the Declaration of Helsinki (revised in 1975) and with CONSORT (Consolidated Standards of Reporting Trials) principles and the regional ethical review board approved the study. All patients provided informed consent.

\section{Investigated parameters:}

Two levels of assessment were conducted, the clinical level and the laboratory lvel investigation which included the histological, immunohistochemical and histomorphometric analyses. These were performed as follow.

\section{Clinical assessment:}

Clinical parameters for evaluation were the capability of bone regeneration of the surgical defect as assesses by comparing the density of bone in three areas of the defect on the coronal cuts, sagittal cuts and on the panoramic view of a cone beam $\mathrm{CT}$ taken immediately postoperative and after three months for each case. Then an average of the density of bone was calculated for three areas in all views to gain a single immediate postoperative and a single three months post operative density of the defect. A ratio of density change (RDC) was calculated by dividing the immediate postoperative density on the three months post operative density for every patient. Moreover, an immediate post operative and three months post operative panoramic (OPG) radiographs were taken for each patient and the defect site was graded using bone Cook's radiographic grading scale ${ }^{[11]}$ which is shown in Table 2.

Table 2: Cook's radiographic scale:

\begin{tabular}{lc}
\hline \multicolumn{1}{c}{ Description } & Grade \\
\hline No change from immediate postoperative appearance & 0 \\
Trace of radiodense material in defect & 1 \\
$\begin{array}{l}\text { Flocculent radiodensity with flecks of calcification } \\
\text { Defect bridged at least one point with } \\
\text { material of nonuniform radiodensity }\end{array}$ & 3 \\
$\begin{array}{l}\text { Defect bridged on both medial and lateral } \\
\text { sides with material of uniform radiodensity, } \\
\text { cut end of the cortex remain visible }\end{array}$ & 4 \\
$\begin{array}{l}\text { Same as Grade 3; at least one of four } \\
\text { cortices is obscured by new bone }\end{array}$ & 5 \\
$\begin{array}{l}\text { Defect bridged by uniform new bone; cut ends } \\
\text { of cortex are no longer distinguishable }\end{array}$ & 6 \\
\hline
\end{tabular}

\section{Laboratory assessment:}

Histological assessment was carried out by comparing the ( $\mathrm{H}$ and $\mathrm{E})$, Masson Goldner trichrome and Giemsa's stained slides. Immunohistochemical examination was performed to gain a descriptive analysis of the cell penetration pattern within the clot. Histomorphometric examination based on immunohistochmesty was performed to attain a quantitative analysis of the cell penetration along the clot by calculating the relative penetration depth (RPD) of every cell type through dividing the penetration length of each cell type by the total length of the clot. Intra group and inter group differences were analyzed.

\section{Statistical analysis:}

Statistics were performed with SPSS software (version 19, IBM Co, USA).

\section{RESULTS}

\section{Clinical results:}

Ten patients were included in the current study with a gender distribution for the study group as two males and three females, and a one male and four females in the control group (statistically insignificant difference: $P$ value of the two tailed Fisher's exact test $=1$ ). Although the mean age of the study group was $31.5 \pm 5.09$ years, it was $28.21 \pm 8.04$ years in the control group (the difference was statistically insignificant: unpaired $\mathrm{t}$ value $=0.773$ and two tailed $P=0.462$ at $\alpha$ level $=0.05$ ).

Table 3 depicts the cause of the surgical defect in the study and the control groups where cyst removal was the main causative factor $(50 \%)$. The $\mathrm{P}$ value of the Fisher's exact test with Freeman-Halton extension was 0.571 (statistically insignificant difference).

Table 3: The cause of the surgical defecct in the study and the control groups:

\begin{tabular}{lccc}
\hline \multicolumn{1}{c}{ The cause } & $\begin{array}{c}\text { The study } \\
\text { group }\end{array}$ & $\begin{array}{c}\text { The control } \\
\text { group }\end{array}$ & Total \\
\hline $\begin{array}{l}\text { Cystic lesion } \\
\begin{array}{l}\text { Compound } \\
\text { odontome }\end{array}\end{array}$ & 3 & 2 & 5 \\
Apicectomy & 2 & 1 & 1 \\
\hline
\end{tabular}

For clinical assessment of the outcome of the present study, Table 4 shows the ratio of density change (RDC) and the Cook's radiographic scaling for both groups. 
Table 4: Clinical parameters of assessment:

\begin{tabular}{|c|c|c|c|c|c|c|c|c|}
\hline & \multirow[t]{2}{*}{$\begin{array}{l}\text { Ratio of density change (RDC) } \\
\qquad\left(\text { mean } \pm \mathrm{SD}^{*}\right)\end{array}$} & \multicolumn{7}{|c|}{$\begin{array}{c}\text { Cook's radiographic grading scale } \\
\text { (number of patients assigned for each scale) }\end{array}$} \\
\hline & & 0 & 1 & 2 & 3 & 4 & 5 & 6 \\
\hline The study group & $7.01 \pm 1.57$ & 0 & 0 & 5 & 0 & 0 & 0 & 0 \\
\hline The control group & $5.67 \pm 0.91$ & 0 & 1 & 4 & 0 & 0 & 0 & 0 \\
\hline statistical test & $\begin{array}{l}\text { unpaired } \mathrm{t}=0.6512 \\
P \text { value }=0.1373 t\end{array}$ & \multicolumn{7}{|c|}{ The $P$ value of the Fisher's exact test $=1 \ddagger$} \\
\hline
\end{tabular}

\section{Laboratory results:}

On the level of the microscopic (laboratory) evaluation, three sub levels were considered as follows.

\section{Histologic results:}

The histologic examination using $\mathrm{H}$ and $\mathrm{E}$ and Masson-Goldner trichrome, and Giemsa's stains revealed three parts within PRF clot which were the red blood cell fraction (RBC), the fibrin clot fraction (FC) and the buffy coat fraction (BC) in between. A looser fibrin clot with more inter fibrous spaces was revealed in the study group specimens than those of the control group which exhibited a denser fibrin clot. Both groups showed more cell concentration within the proximal parts.

\section{Immunohistochemical results:}

The immunohistolochemical examination of the control group showed a concentration of the positively labeled cells in the proximal parts of the clot particularly in the $\mathrm{BC}$ in close proximity to the RBC. With the exception of the CD61- positive cells (platelets) which were distributed throughout the clot albeit also with a proximal tendency, all other labeled cells were almost exclusively concentrated in the $\mathrm{BC}$ portion. These cells were the CD3positive cells (T-lymphocytes), the CD20-positive cells (B-lymphocytes), the CD34-positive cells (stem cells), the CD15-positive cells (neutrophilic granulocytes), and the CD68-positive cells (monocytes).

The study group specimens showed similar results but with a more even distribution of the CD61- positive cells throughout the clot especially the distal portion. The other outstanding difference from the control group was the more extensive distal penetration of the CD15-positive cells (towards the distal parts of the clot) that reached approximately the two thirds of the clot as shown in Figure 1.

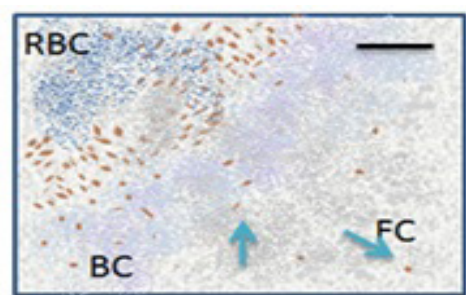

A

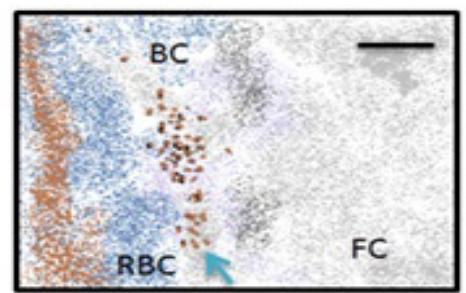

B

Figure 1: The mmunohistochemical image for the detection of the CD15-positive cells (neutrophilic granulocytes) as referred to by the arrows for the study group (A) and for the control group (B). Note the depth of penetration in $\mathrm{A}$ is more distal than in $\mathrm{B}$. 


\section{Histomorphometric results:}

According to the histomorphometric results, the relative penetration depth (RPD) of every cell type was shown in Table 5. Platelets were distributed approximately through out the whole clot within the study and control groups. For the control group the other labeled cells were confined to the proximal one fourth or one third of the clot without any significant difference in between. In the study group;,the other labeled cells were confined in the same zone as that was for the control group except for the neutrophilic granulocytes which had a comparable penetration depth as that of the platelets. distribution pattern within the matrix) and on the tissue regeneration potential.

In the current study, the inclusion and exclusion criteria and the age group targeted were set to allocate a homogenous study population has no healing problems or bone metabolic diseases as far as possible. The surgical defect size was selected to be relatively small and in one jaw (the anterior maxilla was chosen) to allow for more rapid healing and thus more obvious radiographic evaluation. In order to assess the regeneration potential, a radiographic examination using coronal and sagittal cuts in a $\mathrm{CBCT}$ and panoramic views was performed and bone

Table 5: The relative penetration depth (RPD) for each cell type:

\begin{tabular}{|c|c|c|c|c|}
\hline \multirow{2}{*}{ Cell type } & \multicolumn{2}{|c|}{ RPD* } & \multirow{2}{*}{$\begin{array}{l}\text { Inter group difference } \\
\quad \text { (unpaired t test) }\end{array}$} & \multirow{2}{*}{$\begin{array}{l}\text { Significance } \\
\text { at } \alpha=0.05\end{array}$} \\
\hline & $\begin{array}{l}\text { Study group } \\
(\text { mean } \pm \mathrm{SD} \ddagger)\end{array}$ & $\begin{array}{l}\text { Control group } \\
(\text { mean } \pm \mathrm{SD}+)\end{array}$ & & \\
\hline CD3-positive & $0.2 \pm 0.03$ & $0.19 \pm 0.06$ & $\mathrm{t}=0.0372, p=0.7475$ & insignificant \\
\hline CD20-positive & $0.17 \pm 0.01$ & $0.12 \pm 0.09$ & $\mathrm{t}=1.235, \quad p=0.252$ & insignificant \\
\hline CD34-positive & $0.15 \pm 0.01$ & $0.14 \pm 0.04$ & $\mathrm{t}=0.5423, p=0.6024$ & insignificant \\
\hline CD68-positive & $0.22 \pm 0.05$ & $0.21 \pm 0.01$ & $\mathrm{t}=0.4385, p=0.673$ & insignificant \\
\hline CD15-positive & $0.66 \pm 0.14$ & $0.24 \pm 0.02$ & $\mathrm{t}=6.6408, p=0.0002$ & significant \\
\hline CD61-positive & $0.86 \pm 0.23$ & $0.82 \pm 0.04$ & $\mathrm{t}=0.3831, p=0.7116$ & insignificant \\
\hline Intra group differnce & $P<0.001$ !! & $P<0.001$ !! & \multicolumn{2}{|c|}{ One way ANOVA test } \\
\hline Post-hoc Tuky HSD test & \multicolumn{4}{|c|}{$\begin{array}{l}\text { for the study group the CD15-positve cells and CD61-positive cells had a statistically } \\
\text { significant difference from other cells but not in-between, however, for the control group } \\
\text { the CD61-positive cells had a statistically significant difference from other cell types }\end{array}$} \\
\hline
\end{tabular}

To conclude the laboratory results, the study group had a looser fibrin clot with larger inter fiber spaces and deeper penetration of neutrophilic granulocytes than that found in the control group.

\section{DISCUSSION}

Tissue regeneration by a material-induced tissue reaction to fill a surgical defect has gained a wide acceptance ${ }^{[2]}$. Some of the techniques based on that principle require a precultivation and special cell manipulation techniques, a factor led to limiting its clinical application ${ }^{[3]}$. The first wildly applied technique on the clinical level was the platelet rich fibrin $(\mathrm{PRF})^{[5]}$. Choukroun's PRF provides the surgeon with a three dimensional fibrin network contains growth factors that is loaded with platelets, stem cells, momocytes, neutrophilic granulocytes and B- and T- lymphocytes ${ }^{[6,7]}$. Despite the wide use of the $\mathrm{PRF}^{[8-10]}$, the effect of the cumulative centrifugation force on the fibrin properties, the cell distribution pattern, and on regeneration potential on clinical level is unclear. Thus, the current study aimed at evaluating the effect of the cumulative centrifugation force on the characteristics of the PRF (in terms of the fibrin network and the cell density changes were monitored. A laboratory investigation of samples of the same material as used to bridge the defect was carried out on three levels (the histologic, the immunohistochemical, and histomorphometric) to assess the effect of the cumulative centrifugation force on the characteristic of the produced PRF.

The results of the current study showed that the slower but longer-duration centrifugation resulted in a looser fibrin clot with larger inter-fiber spaces and deeper penetration of neutrophilic granulocytes than did the faster but shorter time centrifugation.

Up to this point, the results of the current study confirms those of Ghanaati et al. ${ }^{[2]}$. It worth noting that, tissue regeneration is a complex process depends on the interplay of multiple cells such as stem cells, immune response cells (lymphocytes, neutrophils, macrophages), fibroblast and epithelial cells. The PRF provides all these types of cells including an important cell type in the regenerative process which is the pluripotent hematopoietic stem cells $(\mathrm{HSCs})^{[12-14]}$. B-lymphocytes increase wound healing ${ }^{[15]}$. Platelets have the ability to release a broad spectrum of mediators as cytokines and growth factors (such as platelet derived growth factor (PDGF), transforming growth 
factor $\mathrm{b}$ (TGF-b), platelet derived endothelial growth factor (PDEGF), etc), von Willebrand factor, $P$-selectin, and fibronectin ${ }^{[16]}$. Monocytes migrate into the inflamed area after the influx of neutrophils to transform into macrophages which have an osteogenetic and angiogenetic activity through the expression of various growth factors and bone morphogenic protein-2 (BMP-2) that aid in tissue repair from the early inflammation stage through tissueremodeling stage ${ }^{[17-19]}$. Neutrophilic granulocytes have tissue regenerative properties by facilitating trafficking of monocytes into the wound to phagocytose necrotic and apoptotic cells and aid in the process of revascularization and lymphogenesis ${ }^{[20,21]}$. Perhaps the small diameter of the neutrophilic granulocyte $(8.5-10 \mu \mathrm{m})$ enabled the extensive penetration depth into the clot. The PRF regeneration potential is now unequivocal whether according to the current study or other various previous studies $^{[12-21]}$, however, does the deeper penetration of neutrophilic granulocytes had a positive regeneration effect?

Despite the promising reports about the regenerative capabilities of the neutrophilic granulocytes reported in more than one study ${ }^{[2,20-21]}$, the current studies showed no statistical significant difference of the increased penetration depth of neutrophilic granulocytes as there were no significant changes in bone formation as assessed by Cook's scale or in bone density. That might be attributed to the fact that the tissue regeneration process is a complex process in the wide scope of the word and depends on the interplay of various and multiple cells with a mutual and complex induction process with various inductive cascades. That is why only one cell type as the neutrophils might lack the capability to affect the regeneration potential alone. Therefore, PRF as a second generation platelet concentrate fibrin based cell-loaded matrix had the same regeneration potential on performing either protocols of the current study.

\section{CONCLUSION}

Surgical defects pose a challenge to the surgeon. One of the solutions is the tissue regeneration including PRF, tissue grafting, etc. PRF is a second generation platelet concentrate fibrin-based cell loaded matrix. Despite the various studies revealed the regeneration potential of the PRF, no enough data is available about the effect of the cumulative centrifugation force on that potential. The current study compared two different protocols to produce the PRF. The clinical and the laboratory results exhibited that the slower but longer duration centrifugation resulted in a looser fibrin clot with larger inter fiber spaces and deeper penetration of neutrophilic granulocytes than did the faster but shorter time centrifugation. But that difference failed to impact the regeneration potential on the clinical level. That might be the result of the complex and interactive nature of the regeneration process where one element alone cannot alter the whole process potential.

\section{CONFLICT OF INTEREST}

The authors declare no conflict of interest.

\section{REFERENCES}

1. E. Roddy, M. R. DeBaun, A. Daoud-Gray, Y. P. Yang, and M. J. Gardner, "Treatment of criticalsized bone defects: clinical and tissue engineering perspectives," European Journal of Orthopaedic Surgery and Traumatology, vol. 28, no. 3. Springer Verlag France, pp. 351 -362, 01-Apr-2018.

2. S. Ghanaati et al., "Evaluation of the tissue reaction to a new bilayered collagen matrix in vivo and its translation to the clinic," Biomed. Mater., vol. 6 , no. 1,2011

3. S. Ghanaati et al., "Advanced platelet-rich fibrin: A new concept for cell- Based tissue engineering by means of inflammatory cells," J. Oral Implantol., vol. 40, no. 6, pp. $679-689$, Dec. 2014.

4. B. L. Eppley, W. S. Pietrzak, and M. Blanton, "Platelet-rich plasma: A review of biology and applications in plastic surgery," Plastic and Reconstructive Surgery, vol. 118, no. 6. Plast Reconstr Surg, Nov-2006.

5. D. M. Dohan et al., "Platelet-rich fibrin (PRF): A second-generation platelet concentrate. Part I: Technological concepts and evolution," Oral Surgery, Oral Med. Oral Pathol. Oral Radiol. Endodontology, vol. 101, no. 3, 2006.

6. F. Perut et al., "Preparation method and growth factor content of platelet concentrate influence the osteogenic differentiation of bone marrow stromal cells," Cytotherapy, vol. 15, no. 7, pp. 830-839, Jul. 2013.

7. C. Y. Su, Y. P. Kuo, H. L. Nieh, Y. H. Tseng, and T. Burnouf, "Quantitative assessment of the kinetics of growth factors release from platelet gel," Transfusion, vol. 48, no. 11, pp. 2414-2420, Nov. 2008.

8. Z. Mazor, R. A. Horowitz, M. Del Corso, H. S. Prasad, M. D. Rohrer, and D. M. Dohan Ehrenfest, "Sinus Floor Augmentation With Simultaneous Implant Placement Using Choukroun's PlateletRich Fibrin as the Sole Grafting Material: A Radiologic and Histologic Study at 6 Months," J. Periodontol., vol. 80, no. 12, pp. $2056-2064$, Dec. 2009.

9. F. M. Huang, S. F. Yang, J. H. Zhao and Y. C. Chang, "Platelet rich fibrin increases proliferation 
and differentiation of human dental pulp cells," J. Endod., vol. 36, no. 10, pp. 1628 - 1632, Oct. 2010.

10. L. He, Y. Lin, X. Hu, Y. Zhang, and H. Wu, “A comparative study of platelet-rich fibrin (PRF) and platelet-rich plasma (PRP) on the effect of proliferation and differentiation of rat osteoblasts in vitro," Oral Surgery, Oral Med. Oral Pathol. Oral Radiol. Endodontology, vol. 108, no. 5, pp. $707-713$, Nov. 2009.

11. S. D. Cook, S. L. Salkeld, and L. P. Patron, "Bone defect healing with an osteogenic protein-1 device combined with carboxymethylcellulose," J. Biomed. Mater. Res. - Part B Appl. Biomater., vol. 75 , no. 1 , pp. $137-145$, Oct. 2005.

12. W. F. Lim, T. Inoue-Yokoo, K. S. Tan, M. I. Lai, and D. Sugiyama, "Hematopoietic cell differentiation from embryonic and induced pluripotent stem cells," Stem Cell Research and Therapy, vol. 4, no. 3. Stem Cell Res Ther, 2013.

13. M. Ogawa, A. C. LaRue, and M. Mehrotra, "Hematopoietic stem cells are pluripotent and not just 'hematopoietic," Blood Cells, Mol. Dis., vol. 51, no. 1, pp. 3 - 8, Jun. 2013.

14. V. Nicolaidou et al., "Monocytes induce STAT3 activation in human mesenchymal stem cells to promote osteoblast formation," PLoS One, vol. 7, no. 7, Jul. 2012.

15. D. E. Boyce, W. D. Jones, F. Ruge, K. G. Harding, and K. Moore, "The role of lymphocytes in human dermal wound healing," Br. J. Dermatol., vol. 143, no. 1, pp. $59-65,2000$.

16. C. N. Jenne, R. Urrutia, and P. Kubes, "Platelets: Bridging hemostasis, inflammation and immunity," International Journal of Laboratory Hematology, vol. 35, no. 3. Int J Lab Hematol, pp. $254-261$, Jun - 2013.

17. T. J. Koh and L. A. DiPietro, "Inflammation and wound healing: the role of the macrophage.," Expert reviews in molecular medicine, vol. 13. Expert Rev Mol Med, 2011.

18. R. P. Pirraco, R. L. Reis, and A. P. Marques, "Effect of monocytes/macrophages on the early osteogenic differentiation of hBMSCs," J. Tissue Eng. Regen. Med., vol. 7, no. 5, pp. 392 - 400, May 2013.

19. J. Maciel et al., "Adsorbed fibrinogen enhances production of bone- and angiogenic-related factors by monocytes/macrophages," Tissue Eng. - Part A, vol. 20, no. $1-2$, pp. $250-263$, Jan. 2014.

20. K. W. Tan et al., "Neutrophils contribute to inflammatory lymphangiogenesis by increasing VEGF-A bioavailability and secreting VEGF-D,' Blood, vol. 122, no. 22, pp. 3666 - 3677, 2013.

21. K. Ley, C. Laudanna, M. I. Cybulsky, and S. Nourshargh, "Getting to the site of inflammation: The leukocyte adhesion cascade updated," Nature Reviews Immunology, vol. 7, no. 9. Nature Publishing Group, pp. 678 - 689, Sep-2007. 